\title{
In vivo upregulation of CD95 and CD95L causes synergistic inhibition of angiogenesis by TSP1 peptide and metronomic doxorubicin treatment
}

\author{
AJ Quesadaa ${ }^{1,4}$, T Neliuss ${ }^{2,3,4}$, R Yap ${ }^{2}$, TA Zaichuk ${ }^{2}$, A Alfranca', \\ S Filleur ${ }^{2}$, OV Volpert ${ }^{\star 2}$ and JM Redondo ${ }^{1}$ \\ ${ }^{1}$ Centro Nacional de Investigaciones Cardiovasculares (CNIC) and Centro de \\ Biología Molecular Severo Ochoa (CBMSO), Facultad de Ciencias, \\ Universidad Autónoma de Madrid, Cantoblanco 28049, Madrid, Spain \\ 2 Department of Urology, Northwestern University Feinberg School of Medicine, \\ Chicago, IL, USA \\ ${ }^{3}$ Otto-von-Guericke-University Magdeburg, Department of Urology, Leipziger \\ Straße 44, Magdeburg, Germany \\ 4 These authors contributed equally to the study \\ * Corresponding author: OV Volpert, Northwestern University Feinberg School \\ of Medicine, Department of Urology, Tarry Research Building, Rm 16-761, \\ Chicago, IL 60611, USA. E-mail: olgavolp@ northwestern.edu
}

Received 23.6.04; revised 09.12.04; accepted 24.1.05; published online 01.4.05 Edited by 0 Volpert

\begin{abstract}
Antiangiogenic thrombospondin-1 (TSP1) induces endothelial cell death via a CD95-mediated cascade. We used this signaling pathway, where CD95/Fas is a rate-limiting intermediate, as a target to optimize the efficacy of TSP1 active peptide, DI-TSP. Like TSP1, DI-TSP upregulated endothelial CD95L in vivo. To modulate CD95 levels, we chose chemotherapy agent doxorubicin (DXR). DXR caused sustained upregulation of CD95 in the activated endothelium at 1/ 100 of the maximal tolerated dose. DI-TSP and DXR synergistically induced endothelial apoptosis in vitro, and in vivo, in developing murine vessels. Fas decoy, TSP1 receptor antibody and Pifithrin, a p53 inhibitor, severely decreased apoptosis and restored angiogenesis by DXR-DITSP combination, evidencing critical roles of CD95 and TSP1. Combined therapy synergistically blocked neovascularization and progression of the bladder and prostate carcinoma. Such informed design of a complex antiangiogenic therapy based on the rate-limiting molecular targets is a novel concept, which may yield new approaches to cancer treatment.

Cell Death and Differentiation (2005) 12, 649-658.

doi:10.1038/sj.cdd. 4401615

Published online 1 April 2005
\end{abstract}

Keywords: antiangiogenesis by thrombospondin-1; endothelial cell apoptosis; primary and secondary receptors; CD95 and CD95L; synergy with doxorubicin; drug optimization by design

\section{Abbreviations: TSP1, thrombospondin-1; DXR, doxorubicin}

\section{Introduction}

During oncogenic conversion, the cells undergo angiogenic switch and gain the ability to promote angiogenesis. ${ }^{1}$ Growing tumors rely on the oxygen and nutrients supplied via autonomous capillary network, and disrupting tumor-associated angiogenesis is becoming a promising new strategy to control tumor growth. Among angiogenesis inhibitors, an important place belongs to the naturally occurring peptides, frequently proteolytic fragments of the extracellular matrix proteins, which trigger apoptosis in proliferating but not in quiescent endothelium. ${ }^{2}$ More than 30 natural and synthetic inhibitors are in clinical trials: the lack of toxic effects makes antiangiogenic agents an attractive long-term treatment alternative for traditional chemotherapy; however, their high cost and relatively low specific activity present a challenge (Kerbel and Folkman ${ }^{2}$ and http://clinicaltrials.gov).

Thrombospondin-1 (TSP1) is an angiogenesis inhibitor, ${ }^{3}$ a multidomain protein that binds a variety of receptors, including integrins, CD36, integrin-associated protein, heparan sulfate proteoglycans and more. ${ }^{4}$ TSP1 blocks angiogenesis via CD36, a receptor constitutively expressed in a subset of endothelial cells. ${ }^{5-7}$ The ability to bind multiple receptors complicates the therapeutic use of nascent TSP1. This obstacle have been circumvented by introducing minimal antiangiogenic fragments of TSP $1 .{ }^{8}$ One of them, DI-TSP is active at nanomolar concentrations that interacts with CD36 and causes endothelial cell apoptosis via the same signaling cascade $^{9}$ (Zaichuk and Volpert, unpublished observations) while lacking undesirable effects due to binding receptors other than CD36. ${ }^{8,9}$ This is a C-ter capped nonapeptide based on the GVITRIR sequence from the second properdin repeat, containing an L- to D-isoleucyl substitution. ${ }^{9}$ TSP1, or DI-TSP binding to CD36 triggers signaling cascade that begins with recruitment of Src-related kinase $p 59^{f y n}$ and ultimately causes endothelial cells apoptosis. ${ }^{10}$ TSP1 selectively destroys proliferating, but not quiescent endothelium: our recent studies attribute this selectivity to the CD36-dependent upregulation of the cell surface CD95L, a ligand for the death receptor CD95/Fas. ${ }^{11}$ Binding of CD95, a member of the TNF receptor superfamily to its ligand ${ }^{12-16}$ triggers apoptotic response via an exhaustively studied pathway that involves formation of the intracellular death-inducing signaling complexes (DISCs). ${ }^{17}$ CD95 itself is constitutively low on the resting endothelial cells and its surface expression is induced by proangiogenic factors such as vascular endothelial growth factor (VEGF), basic fibroblast growth factor (bFGF) or interleukin-8 (IL-8). Thus, CD95/CD95L-mediated apoptosis can only occur in the endothelial cells exposed to both angiogenic inhibitors and stimuli.

Proapoptotic and antiangiogenic effect of TSP1 and peptide mimetics is restricted by the availability of endothelial CD95. CD95 presentation could be improved with increased stimulation by inducers of angiogenesis, however it would cause concomitant increase in survival signals. Seeking other ways to augment an efficacy of DI-TSP, we chose doxorubicin (DXR), a genotoxic agent used for the chemotherapy of 
human leukemias, lymphomas, breast and prostate cancer. DXR at high doses increases surface CD95 and triggers apoptosis in proliferating endothelium. ${ }^{18}$ Interestingly, although at these doses, DXR induced apoptosis independent of CD95/CD95L interactions, the upregulated CD95 remains functional and CD95-dependent apoptotic cascade can be triggered with the activating monoclonal antibodies in DXRtreated cells but not in untreated control population. ${ }^{18}$

Here we show that at low concentrations, DXR resulted in sustained upregulation of the endothelial CD95 without noticeable cytotoxic effects towards endothelial or cancer cells. The increase in the endothelial CD95 by low DXR doses significantly improved the antiangiogenic and antitumor efficacy of DI-TSP. The use of the low DXR doses administered continuously at regular short intervals (metronomic chemotherapy) as opposed to administration of several bolus doses may lower cardiac and liver toxicity commonly associated with genotoxic DXR treatment, as well as the emergence of multidrug resistance. ${ }^{19-21}$ Indeed, we showed that DXR at doses 100 times lower then those effectively used as monotherapy ${ }^{19,22,23}$ significantly upregulated endothelial CD95 in vitro and in vivo: this DXR-generated CD95 increase contributed to the augmented endothelial cell apoptosis by DITSP leading to the synergistic reduction of angiogenesis and tumor growth while avoiding toxic side effects of the high therapeutic doses.

\section{Results}

\section{Low DXR doses augmented CD95/Fas surface expression by endothelial cells}

In vitro DXR at concentrations equivalent to the maximum tolerated therapeutic doses (MTD, 250 ng/ml) ${ }^{23,24}$ induces upregulation of a functional cell surface CD95 by the large vessel endothelium (HUVEC). ${ }^{18}$ Here, we tested whether DXR doses below MTD (50-200 $\mathrm{ng} / \mathrm{ml})$ were sufficient to upregulate endothelial CD95. We analyzed the effect of DXR between 50 and $500 \mathrm{ng} / \mathrm{ml}$ on apoptosis and CD95 presentation of HUVECs and microvascular endothelial cells (HMVECs) cultured in full serum. DXR induced significant endothelial cell apoptosis only at $500 \mathrm{ng} / \mathrm{ml}$. Although lower DXR concentrations failed to trigger apoptosis in HUVECs and HMVECs, or to delay the cell cycle progression (Figure $1 \mathrm{a}, \mathrm{b}$ and supplementary Figure 1a), quantitative flow cytometry analysis showed a substantial increase of the surface CD95 (Figure 1c, d and data not shown). Baseline fluorescence of the cells stained with the secondary antibody alone was not affected by the isotype control IgG. Thus, DXR significantly increased surface CD95 at doses at least 10 times lower than those required to induce endothelial cell apoptosis in vitro. ${ }^{18}$

\section{DI-TSP upregulated CD95L expression and induced HMVECs apoptosis in synergy with DXR}

TSP1 increases mRNA and surface CD95L in the endothelial cells via signaling cascade mediated by its antiangiogenic receptor, CD36. ${ }^{10,11,25}$ Since HUVECs are CD36 negative, ${ }^{6}$ we examined the ability of DI-TSP, a short peptide derivative

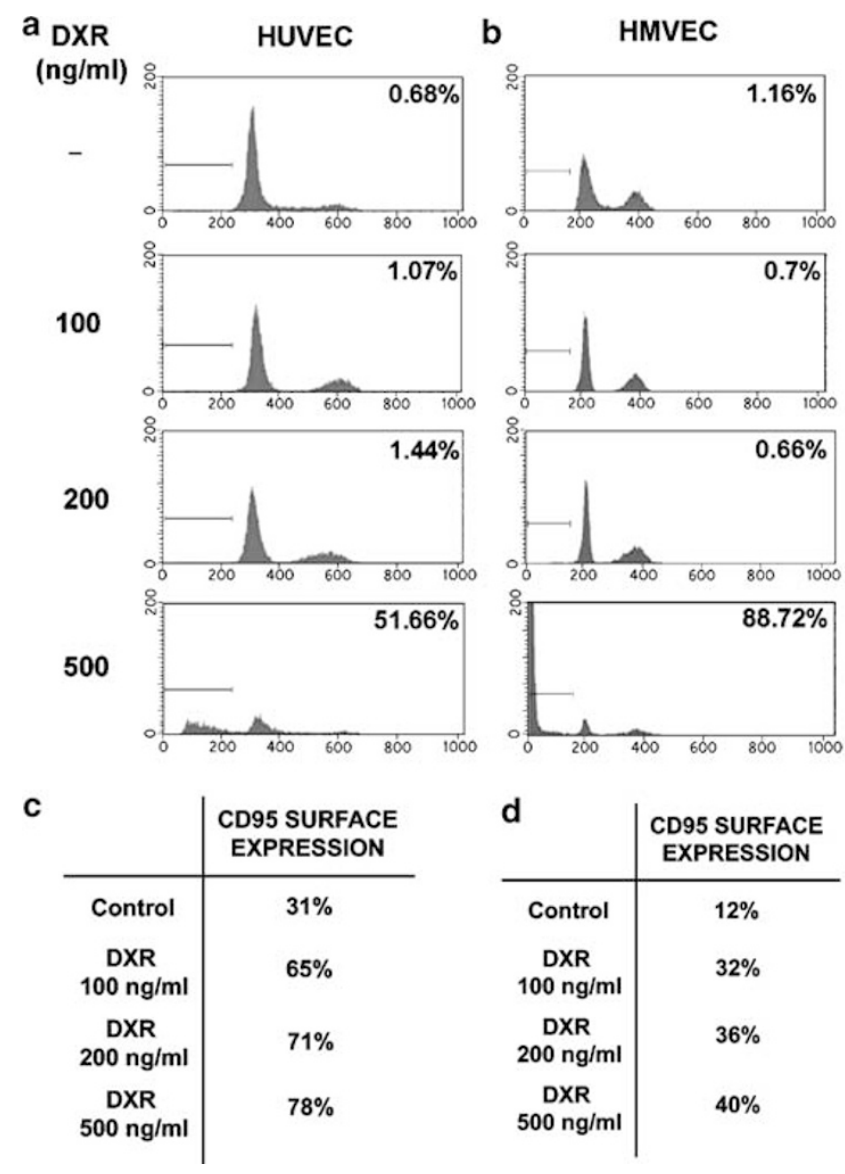

Figure 1 Cell cycle and CD95 surface levels in DXR-treated endothelial cells. HUVECs (a) and HMVECs (b) were treated $48 \mathrm{~h}$ with the indicated DXR concentrations, stained with propidium iodide and DNA content analyzed by flow cytometry. Note the significant percentage of the apoptotic cells in the presence of $500 \mathrm{ng} / \mathrm{ml} \mathrm{DXR}$. (c, d) Quantification of flow cytometry analysis of surface CD95 expression in DXR-treated HUVECs (c) or HMVECs (d). Note the considerable increase in CD95-positive cell population at lower DXR concentrations. Control values reflect the percentage of FITC-positive cells after incubation with the FITC-conjugated secondary antibody alone

of TSP1, to induce similar events only in the CD36-positive HMVECs. ${ }^{5,6}$ Treatment $(24 \mathrm{~h})$ with DI-TSP at antiangiogenic, proapoptotic concentration $(10 \mathrm{nM})$ resulted in pronounced upregulation of CD95L that was preserved in the presence of DXR (Figure 2a).

We then combined DXR doses, which in a single treatment increased endothelial CD95 surface expression but not apoptosis, with DI-TSP and tested their ability to cooperatively induce apoptosis in proliferating HMVECs. Importantly, up to $70 \%$ cells displayed signs of apoptosis in HMVEC population treated with DI-TSP/DXR combination. Single agents produced little increase in cell death compared to the baseline control levels as was determined by TUNEL (Figure $2 \mathrm{~b}$ ) and by flow cytometry analysis of DNA content (data not shown). Thus, DXR and DI-TSP acted in synergy (see below) to induce HMVEC apoptosis in vitro. As we anticipated, this synergistic induction of apoptosis was CD95 and CD95L dependent as CD95 decoy receptor diminished apoptosis rate to $\sim 9.5 \%$ (Figure 2b). Basal levels of CD95L combined with the modest 
a

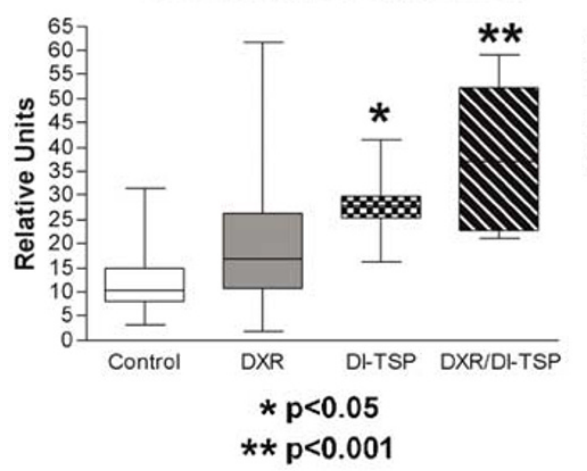

b ApopTag

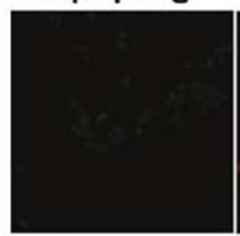

CD31

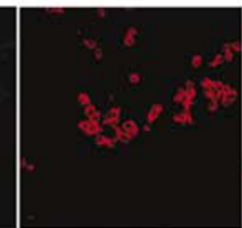

Merge
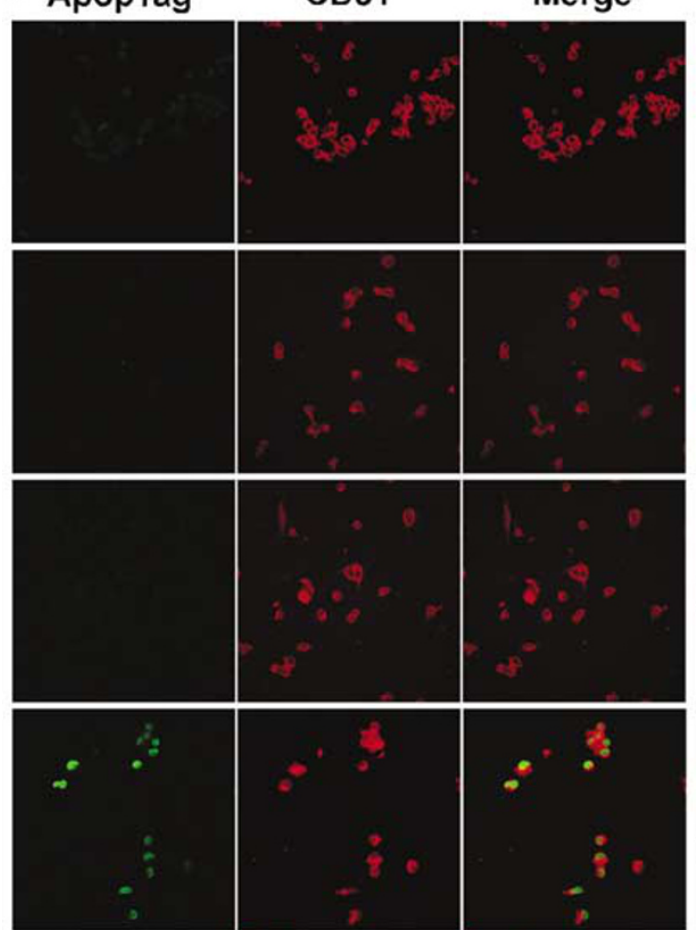

DXR
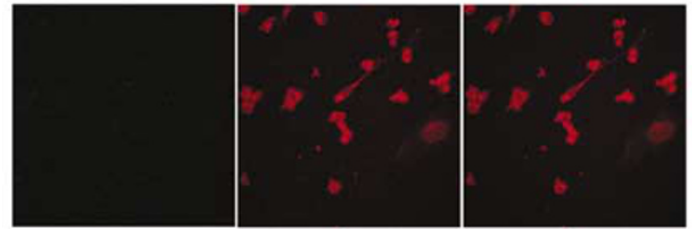

DXR

DI-TSP

Fas D

Figure 2 Combination of low doses of DXR and DI-TSP induce CD95, CD95L and apoptosis in cultured HMVECs. (a) HMVECs were treated $24 \mathrm{~h}$ with $10 \mathrm{nM}$ DI-TSP and/or $100 \mathrm{ng} / \mathrm{ml}$ DXR as indicated and stained in situ for CD95L and CD31 endothelial cell marker. CD95L expression was measured on digital images using LaserPix software and normalized against CD31 levels. Note the upregulation of CD95L expression in DI-TSP-treated cells. (b) Apoptosis was analyzed by in situ TUNEL assay. DI-TSP/DXR treatment was repeated in the presence of soluble CD95 decoy receptor (FasD). Cell population was counterstained with CD31 (red). Each field includes roughly equal number of cells. Note high apoptosis levels in the presence of DI-TSP/DXR combination and reversal by CD95 decoy

CD95 increase due to the low dose of DI-TSP were insufficient to induce apoptosis at significant levels in the cells cultured in serum-rich medium (Figure 2a,b).

\section{DXR and DI-TSP treatment upregulated CD95, CD95L on remodeling capillaries and increased apoptosis in vivo}

We next sought to determine whether DI-TSP and low DXR doses cooperatively block angiogenesis in vivo. We chose $0.2 \mathrm{mg} / \mathrm{kg} / 5$ days DXR regimen for the in vivo treatment: it is 25 times lower than the bolus therapeutic dose of DXR conventionally used to block tumor growth in mouse models and 100 times lower than maximum tolerated dose (MTD). ${ }^{19,22,23}$ We used Matrigel plug model where murine vessels develop subcutaneously towards and into the extracellular matrix plug. This model allows the study of developing blood vessels over time, while excluding interference by the tumor cells. Detection of DXR-dependent increase in CD95/ Fas proved difficult, as the plugs were supplemented with the excess of exogenous proangiogenic factors (VEGF or bFGF), which are known to induce endothelial CD95. ${ }^{11}$ Seeking conditions that would allow unequivocal demonstration of in vivo CD95 upregulation by the chosen DXR dose, we performed the assay using Matrigel plugs lacking exogenous VEGF and heparin. We were able to detect at least a 10-fold increase in CD95 staining in the presence of DXR $(0.2 \mathrm{mg} / \mathrm{kg})$ (Figure $3 a, b)$. In standard Matrigel supplemented with proangiogenic factors, we were able to detect CD95L in the new capillaries treated with either DI-TSP or DI-TSP/DXR combination (Figure 3c). Thus, endothelial cells of the remodeling capillaries in vivo express higher levels of CD95 in response to DXR and increase CD95L in response to DITSP.

We were able to detect an apparent increase in the apoptotic endothelial cell population in response to DI-TSP/ DXR combination, where both CD95 and CD95L were upregulated (Figure $3 \mathrm{~d}$ ). Interestingly, the expression of CD31 (PECAM-1) by the endothelial cells undergoing apoptosis was markedly lower (see Discussion); however, localization of CD31 to the apoptotic cells was evident. (Figure 3d, inset). Finally, DXR alone caused no significant change in MVD in the Matrigel plugs compared to the untreated control. DI-TSP at $1 \mathrm{mg} / \mathrm{kg}$ caused a 1.4 decrease in the median vascular density $(P<0.06)$, and the combination treatment produced a striking 2.9 -fold decrease $(P<0.002)$.

\section{DI-TSP and DXR at low doses delayed tumor progression and diminished microvessel density}

In order to investigate whether DXR and DI-TSP combination would affect tumor progression and tumor-associated angiogenesis, we used xenograft model of the human carcinoma of the bladder 253J B-V, a highly aggressive cell line completely lacking secreted TSP1. ${ }^{26}$ Animals that received DI-TSP/DXR combination treatment showed a significant delay in tumor onset compared to the single treatments and to the control vehicle. This delay was followed by tumor stabilization (Figure 4a). When combined DI-TSP/DXR treatment was applied to a less aggressive prostate carcinoma cell line $\mathrm{LNCaP}$, the results were even more striking (Figure 4b). While low dose of DXR alone had no significant effect on the tumor progression and DI-TSP caused only modest several days' delay, DI-TSP/DXR combination treatment resulted in the 


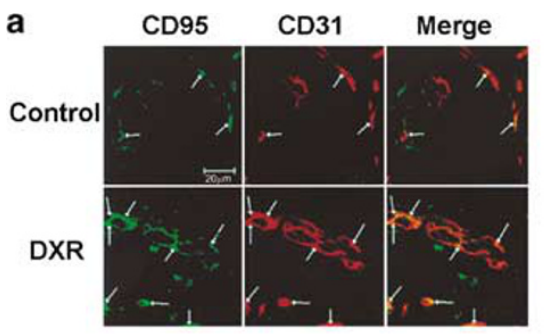

b $\widehat{g}$

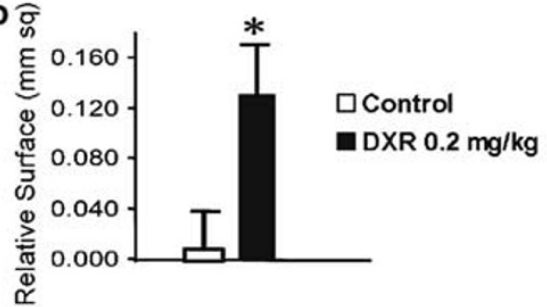

C
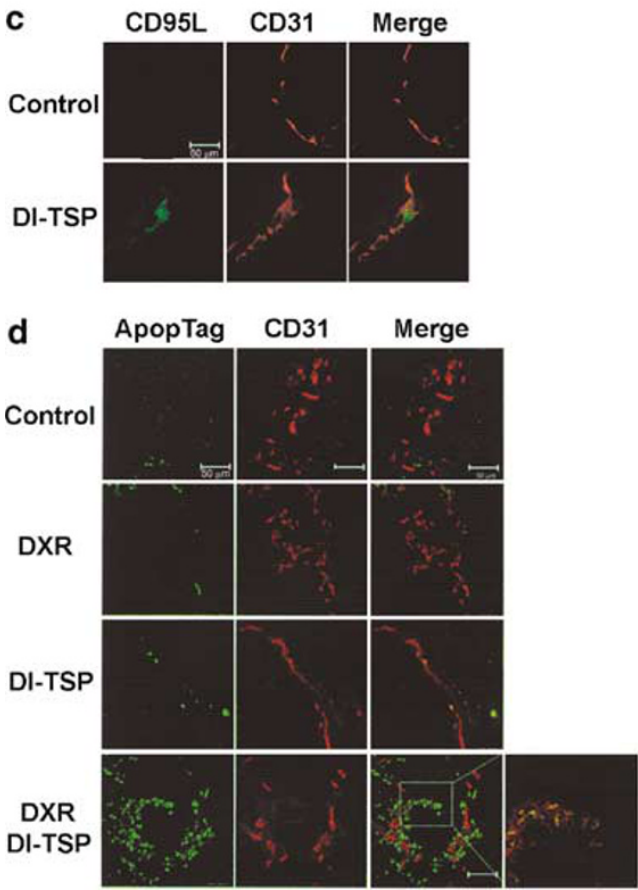

Figure 3 The effect of DI-TSP and DXR on CD95 and CD95L expression in newly formed capillaries in the Matrigel plugs in vivo. (a) CD95 levels (green) evaluated in the DXR-treated neovessels (red) in the absence of stimulatory VEGF in the Matrigel plugs. Arrows indicate CD95-positive endothelial structures. (b) Quantitative analysis of the experiment in (a). ${ }^{*} P \leqslant 0.002$. (c) Mice were given Matrigel plugs containing VEGF, treated as indicated and CD95L (green) assessed by immunofluorescence. Endothelial structures are visualized by CD31 staining (red). (d) Similarly treated Matrigel plugs were stained for apoptosis (TUNEL, green). Note the significant increase in the endothelial cell death by DITSP/DXR combination. CD31 (red) was used as an endothelial cell marker. Also, note weaker CD31 staining of the apoptotic endothelial structures (inset)

eight-fold reduction of the tumor volume and a considerable progression delay. Interactive coefficient I for DI-TSP calculated using modified isobologram analysis ${ }^{27}$ was $>1(\sim 70$ and 50 for the bladder and prostate cancer models, respectively), indicating strong synergy. Microvessel density (MVD) of these tumors followed the same pattern in that it was
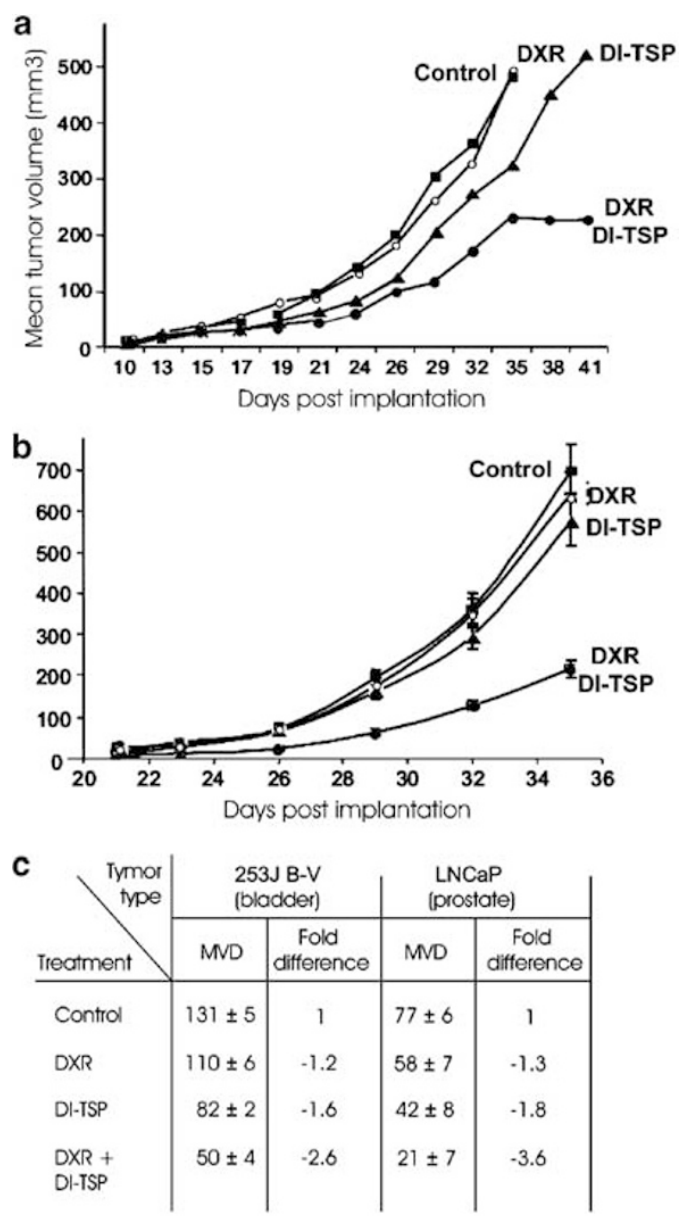

d

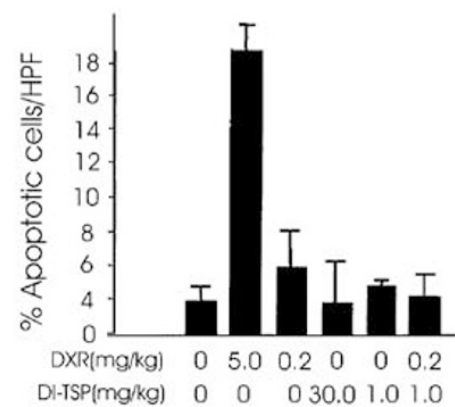

Figure 4 Combination of low DXR doses with DI-TSP treatment augments the delays in tumor progression and further reduces tumor vascularity. Nude mice were given subcutaneous bladder carcinoma tumors and treated with control saline or DI-TSP $\pm D X R$. (a) The effect of DI-TSP/DXR combination on progression of bladder carcinoma xenografts. (b) The effect of the same treatment on progression of prostate carcinoma xenografts. (c) Tumor vascularity in 253J B-v and LNCaP tumors was assessed by CD31 staining of tissue sections and quantified using ImageQuant software. Microvessel counts and fold reduction are shown. All the differences are significant $(P<0.05)$. (d) Apoptosis in the cardiac muscle measured by in situ TUNEL assay

significantly lower in the tumors extracted from mice treated with the combination therapy, DI-TSP/DXR (Figure 4c and supplementary Figure 1b). DI-TSP alone produced a mild decrease in the tumor MVD and size while DXR alone had no significant effect (Figure 4c and supplementary Figure 1b). Interestingly, higher DXR doses resulted in a different pattern 
of tumor progression: after the initial delay, tumors in the groups treated with DXR alone or in combination with DI-TSP grew at a faster rate (see Discussion).

Bolus DXR administration at MTD causes apoptosis in multiple organs, often resulting in heart and liver failure. We measured apoptosis in the hearts of mice after 2 weeks of DXR boli, metronomic DXR and DI-TSP/metronomic DXR combination. MTD resulted in $>$ four-fold higher apoptosis in the cardiac muscle compared to the control vehicle, no measurable increase was observed with DI-TSP/DXR combination (Figure $4 d$ ). Animals treated with MTD lost $\sim 15 \%$ of their body weight while combined treatment caused no significant weight loss (data not shown).

\section{DXR/DI-TSP combination induced CD95, CD95L and apoptosis in tumor vasculature}

Compared to the subcutaneous Matrigel plugs, the environment of the tumor vasculature is extremely complex. Thus, it was nonevident that the effect of DI-TSP and DXR on CD95/ CD95L expression would remain the same. Nevertheless, in mice treated with DXR/DI-TSP combination, we detected profound upregulation of CD95L in tumor vessels in the presence of DI-TSP alone or in combination with DXR (Figure 5a, right). An increase in CD95 was less evident due to the high background levels caused by tumor-derived proangiogenic factor(s); however, quantitative evaluation of immunostained tumor sections showed increased number of the CD95-positive endothelial structures (Figure 5a, left).

At-a-glance, evaluation of the apoptosis levels in tumor xenografts showed a dramatic increase in total numbers of TUNEL-positive cells in the tumors treated with DI-TSP/DXR

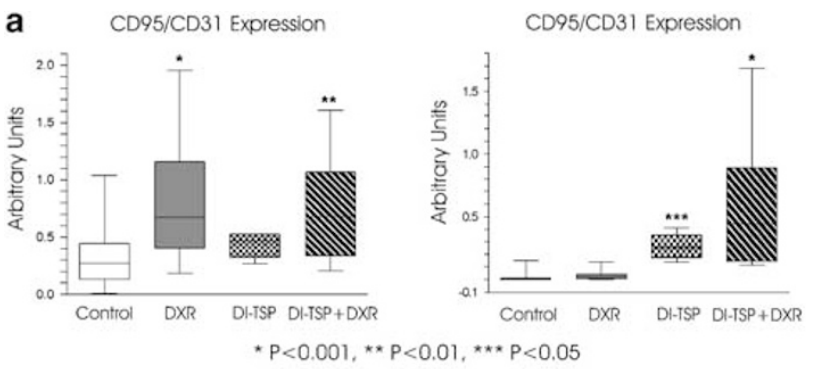

b

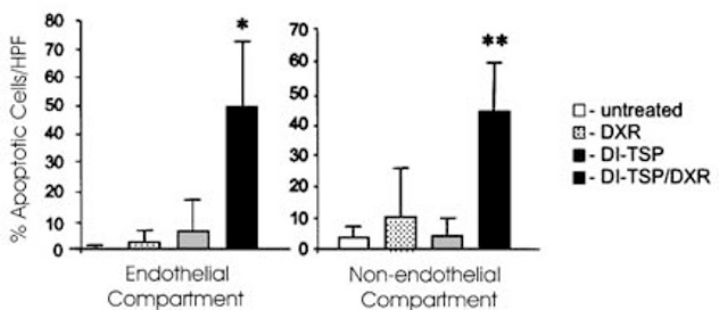

Figure 5 Combination of low DXR doses with DI-TSP treatment augments CD95 and CD95L expression in tumor endothelial cells and increase apoptosis in the tumors. (a) CD31-positive structures (blood vessels) within tumors subjected to the indicated treatments were evaluated for the expression of CD95 (left) and CD95L (right). Note the significant upregulation of CD95 by DXR and of CD95L by DI-TSP. (b) Apoptosis in the endothelial (left panel) and in the tumor cell compartments (right) are shown separately. ${ }^{*} P \leqslant 0.002,{ }^{\star *} P \leqslant 0.003$ combination compared to the single treatments and to the sham-treated control (Figure 5b). To distinguish between the antiangiogenic and tumoricidal effects of the treatments, we performed quantitative analysis of the apoptotic cell populations separately in the endothelial (CD31-positive) and nonendothelial (CD31-negative, tumor and inflammatory cells) compartments of the tumors. The combined therapy caused striking increase in the apoptosis in both endothelial and nonendothelial compartments of the tumor, while the same dose of DXR alone failed to affect endothelial cells and had very modest effect on the tumor cells, suggesting that tumor cell apoptosis was largely due to the restrictions posed by the lack of nourishing vasculature.

\section{Angiogenesis blockade by DI-TSP/DXR combination was mediated by TSP1 receptor and required $C D 95 / C D 95 L$ death cascade}

The synergy between DXR and DI-TSP in vivo was dependent on the signal generated by CD95L binding to CD95/Fas on the microvascular endothelium. Soluble CD95/Fas-Fc receptor (Fas decoy) significantly attenuated MVD reduction by the combined treatment and inhibited endothelial cell apoptosis in Matrigel plug assay (Figure $6 a-c)$. The inhibitory activity of the combination therapy was clearly associated with TSP1 signaling cascade. Neutralizing antibody against TSP1 receptor CD36 were previously shown to inhibit TSPmediated induction of CD95L and apoptosis. ${ }^{10,11}$ The same antibody completely abrogated endothelial cell apoptosis and antiangiogenesis by DI-TSP/DXR combination (Figure 6a-c). Since CD95 upregulation by DXR is p53-dependent ${ }^{18}$ while apoptosis by DI-TSP is not, ${ }^{10}$ we used Pifithrin- $\alpha$ (PFT- $\alpha$ ), an inhibitor of p53-dependent transcription, ${ }^{28}$ in order to eliminate DXR induction of CD95 mRNA and protein. Predictably, PFT $-\alpha$ relieved the effect of DI-TSP/DXR on both microvessel counts and endothelial cell.

\section{Discussion}

Angiogenesis inhibitors are gaining their due place among cancer treatments. Since their primary target is normal, genetically stable endothelium, and not the labile tumor cells, the selection pressure is less likely to evoke resistance. Antiangiogenics target no particular tumor lineage and thus can be applied to a variety of tumors. Such therapies delay progression of highly and of poorly vascularized tumors ${ }^{29}$ and the reports from the preclinical studies hold promise. ${ }^{30}$ However, some caveats already emerged, for example, persistent hypoxia due to angiogenesis blockade causes selection of the tumor cells with higher resistance to the stress-induced apoptosis. ${ }^{2}$ These findings imply the importance of maximizing the efficacy of inhibitors in order to limit the time of exposure to acute hypoxia. Here, we developed a rational approach to maximize the efficacy of angiogenesis inhibitor, TSP1 based on its molecular mechanism of action.

The first steps to make TSP1 suitable for clinical use have already been taken. Although nascent protein strongly inhibits angiogenesis, ${ }^{31}$ its ability to bind multiple receptors is disconcerting, since some of these receptors, including 


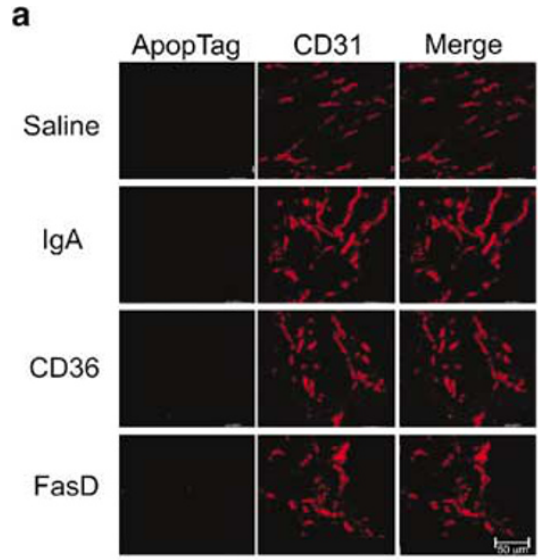

Control

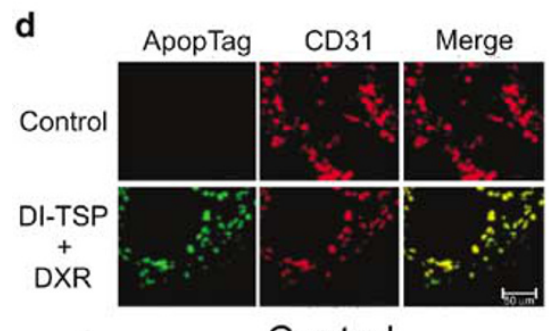

Control

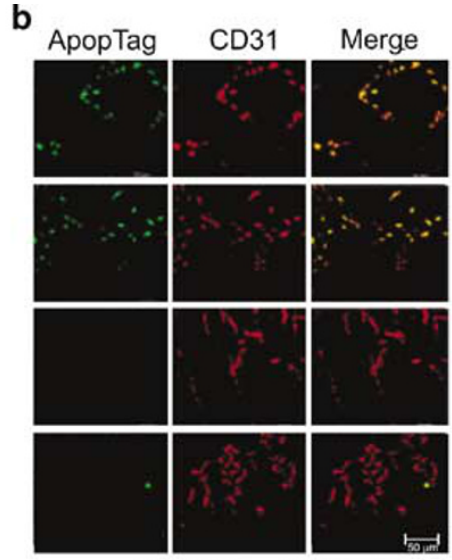

DI-TSP + DXR

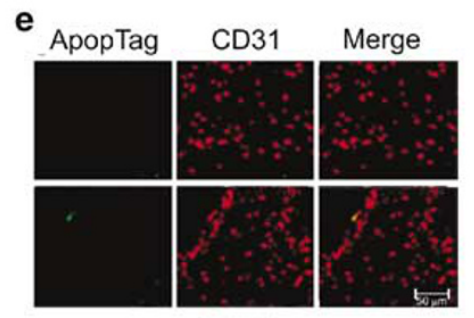

PFT- $\alpha$
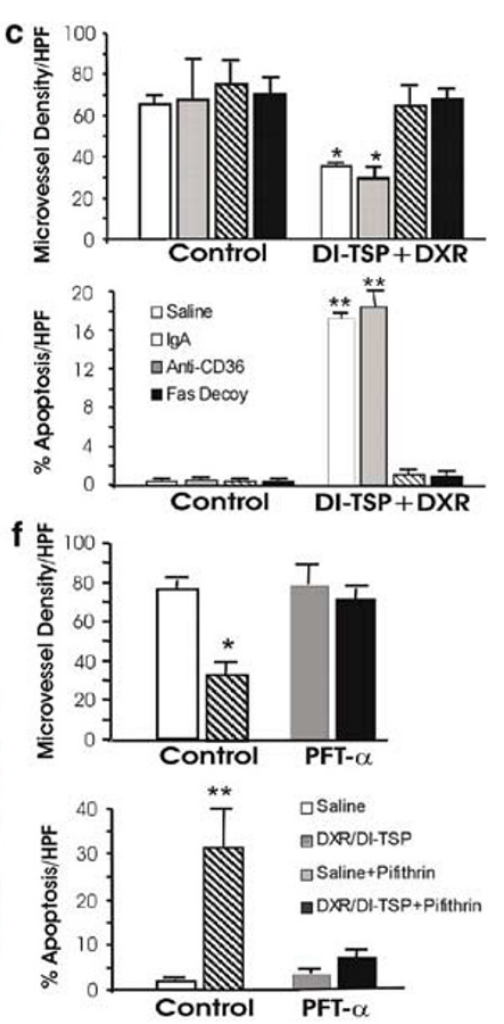

Figure 6 The role of CD35, CD95 and p53 in the anti-angiogenesis and apoptosis by DI-TSP/DXR combination. (a-c) Mice given subcutaneous Matrigel plugs containing VEGF were treated with vehicle PBS (a) or DI-TSP/DXR combination (b). CD36-neutralizing antibodies, anti-mouse IgA isotype control antibody and CD95FC decoy receptor were added where indicated. Note that high apoptosis levels in treated plugs versus control were decreased visibly by either CD36 antibody or by CD95 decoy, but not by isotype control antibody. (c) Quantitative analysis of the experiment shown in (a, b). $\square$ PBS, $\square \operatorname{lgA}, \mathbb{N}$ anti-CD36, $\square$ CD95 decoy. ${ }^{*} P \leqslant 0.004$, ${ }^{* \star} P \leqslant 0.002$ (d-f) Mice with subcutaneous Matrigel plugs were treated with control PBS (d and $\mathbf{e}$, upper panel) or DI-TSP/DXR combination (d and $\mathbf{e}$, lower panels), alone (d) or with PFT- $\alpha$ (e), an inhibitor of p53-dependent transcription. (f) Quantification of the experiment in (d, e). $\square$ PBS, $\mathbb{D}$ DI-TSP/DXR, $\square$ PFT $\alpha, \mathbf{D}$ DI-TSP/ $\mathrm{DXR}+\mathrm{PFT}-\alpha,{ }^{*} P \leqslant 0.001,{ }^{\star *} P \leqslant 0.0003$

integrin-associated protein, mediate functions incompatible with antiangiogenic therapy. ${ }^{32}$ Minimal antiangiogenic fragments of TSP1 mapping to the type I repeats and preprocollagen homology domain share GVTRIR consensus sequence ${ }^{8,33}$ and block angiogenesis in vitro and in vivo by inducing endothelial cell apoptosis that occurs upon binding to CD36, a cell surface receptor expressed predominantly on the microvascular endothelium. ${ }^{5,6,10}$ DI-TSP, a modified GVTRIR-based peptide, blocks endothelial cell functions, angiogenesis and tumor growth with relatively high specific activity. ${ }^{34}$ In this study, ${ }^{34}$ the dose of DI-TSP that inhibited tumor growth by $70 \%$ and diminished MVD by $50 \%$ was $30 \mathrm{mg} / \mathrm{kg}$ twice a day, 60 times higher than the dose used in this report. A total of $1 \mathrm{mg} / \mathrm{kg} / \mathrm{twice}$ daily $(2 \mathrm{mg} / \mathrm{kg})$ caused a modest $30 \%$ decrease in tumor volume, similar to what we observe in a subcutaneous model (Figure 4). These peptides are now in Phase 1 and 2 clinical trials with the evidence of favorable outcomes mounting (see June 2003 press release from Abbott Labs). Another option is modulation of the primary receptor or TSP1, CD36 that can be achieved by treatment with the ligands of the nuclear receptor PPAR- $\gamma{ }^{35}$

The ability of TSP1/DI-TSP to selectively eliminate remodeling endothelium is based on the secondary receptormediated signaling cascade where TSP-dependent expression of CD95L triggers apoptosis of the CD95/Fas-positive endothelial cells. ${ }^{11}$ CD95 expression is a function of remodeling induced by angiogenic stimuli (VEGF, bFGF, etc.), it is independent of TSP1, and limits its efficacy. ${ }^{11,36}$

Seeking factors to improve antiangiogenic effect of DI-TSP, we examined DXR, a common chemotherapy agent that increase CD95/Fas presentation by the activated endothelium. We recently showed that DXR at $500 \mathrm{ng} / \mathrm{ml}$ induces apoptosis in proliferating endothelial cells, and induces p53dependent increase in the mRNA and cell surface expression levels of CD95. Although this increase in the expression of CD95 was not involved in the signaling cascade that led to caspase activation and endothelial cell apoptosis by DXR, CD95 itself was functionally active and capable of inducing cell death upon stimulation with agonistic CD95 antibodies, showing that the required molecular machinery remained functional. ${ }^{18}$ Here, we showed that DXR concentrations as low as $50 \mathrm{ng} / \mathrm{ml}$ still increased CD95, but caused no significant apoptosis in EC nor in cancer cells. These noncytotoxic concentrations augmented in vitro EC sensitivity to apoptosis by DI-TSP via CD95 cascade. Modified isobologram analy$\mathrm{sis}^{27}$ yielded interaction coefficient of $>50$, clearly pointing to synergy.

When DISC formation is followed by caspase activation, CD95/Fas is internalized to be recycled via clathrin-coated pits and caveolae (reviewed in Peter and $\mathrm{Krammer}^{37}$ ), similar 
events are involved in the CD95 trafficking and surface upregulation by the endothelial cells in response to angiogenic stimuli. ${ }^{11}$ Thus, the intracellular pool of CD95/Fas protein clearly limits its availability for the surface exposure by inducer-stimulated endothelium. Conversely, DXR treatment triggers a sustained, p53-dependent upregulation of CD95 mRNA and consequently increases the total CD95 available for the surface presentation and ligand interaction.

On the other hand, strong and sustained p53 expression in the DXR-treated endothelial cells may render these cells more susceptible to apoptosis by TSP1/DI-TSP. ${ }^{38,39}$ We found that DXR-induced p53 increased APAF-1, a component of the mitochondrial apoptotic pathway ${ }^{40}$ and PUMA expression levels (see supplementary Figure 2), ${ }^{41}$ which, in turn could sensitize endothelial cells to the death by CD95 ligation. Indeed, dramatic reduction of antiangiogenesis by the DXR/ DI-TSP combination by $\mathrm{p} 53$ inhibitor, PFT- $\alpha$ pointed to a major contribution of the p53 to their synergy.

On the other hand, hypoxic conditions due to the antiangiogenic arm of the treatment altered the tumor cells responsiveness to the low doses of DXR. Hypoxia severely diminished the increase of p21 in response to DXR while significantly increasing the proportion of cells arrested in G2/ $M$ phase and somewhat increasing apoptosis (see supplementary Figure 2). Although p21 predominantly serves as an inhibitor of cell proliferation, it also plays an important role in drug-induced tumor suppression. Nevertheless, a number of recent studies have shown that p21 (WAF1/Cip1) can assume both pro- or antiapoptotic functions in response to antitumor agents depending on cell type and cellular context. ${ }^{42}$

Continuous application of the low doses of chemotherapy agents (metronomic dosing) was proposed as a replacement for the discontinuous use of the same agents at MTD in a 'front-loading' manner. Such treatments are thought to minimize the long-term tumor burden by acting as antiangiogenic agents as well as by reducing the selection for treatment-resistant population of the tumor cells. ${ }^{43}$ That is one possible explanation of how higher DXR doses, although upregulating CD95 expression on the endothelial cells, trigger different tumor progression pattern, in which tumor development, albeit initially slower, later gained momentum. On the other hand, rapid regrowth of the tumor in the periods between bolus doses of chemotherapy causes higher levels of secreted growth factors and thus more vigorous angiogenesis. ${ }^{43}$ In addition, angiogenesis inhibitors, such as TNP470 minocyclin and DC101, have been shown to improve the efficacy of chemotherapy agents, like cisplatin, cylophosphamide and paclitaxel by improving small molecule delivery to the tumor mass due to reduced interstitial pressure. ${ }^{44,45}$

Here, we investigate the combination of DI-TSP, an antiangiogenic agent, and of metronomic dosage of a chemotherapy agent DXR that have cooperative effect in reducing tumor angiogenesis. DXR greatly increased the ability of DI-TSP to block angiogenesis and tumor growth in vivo. The dose of DXR used in our treatment regimens was 25-100 lower than MTD commonly used in mouse tumor models. ${ }^{22,23,46}$ Interaction index was far greater than 1, clearly indicating synergy, which was critically dependent on DI-TSP signaling intermediates CD36 and CD95L. Combined DI-TSP/ DXR inhibition of angiogenesis was relieved in the presence of
anti-CD36-blocking antibodies. Moreover, this synergy was based on CD95-dependent signaling events because the combined antiangiogenic effect and enhanced endothelial cell apoptosis in vivo were eliminated by soluble CD95 decoy. Apparently low doses of DXR rendered tumor cells more sensitive to the stress-induced apoptosis due to the vascular reduction by DI-TSP by upregulating proapoptotic genes and increasing G2/M arrest. Interestingly, higher CD95/CD95L expression by remodeling capillaries in the presence of DITSP and DXR associated with apoptosis and antiangiogenesis invariably correlated with a dramatic decrease in CD31 levels. CD31 ligation has been implicated in survival signals such as Akt-dependent increase of Bcl-2 A1 and Bcl-X. ${ }^{47,48}$ It is also possible that CD31 was decreased due to dissociation of the cells within affected vessels: strong lateral expression of CD31 is typical for the endothelial cells forming contacts and is thought to play a role in vascular permeability. ${ }^{49}$ In this case, decrease in CD31 is likely treatment-related and further facilitates apoptosis by DI-TSP/DXR combination.

One obvious advantage provided by the metronomic dosing of chemotherapy agents is their decreased toxic effect towards noncancerous cells. ${ }^{50}$ Indeed apoptosis levels induced by metronomic DXR treatment in experimental animals were considerably lower compared to MTD treatment and had no significant effect on background apoptosis levels in the heart and liver.

The increase in CD95/Fas expression by the resting capillaries and large vessels was an important concern as such upregulation could compromise the integrity of preexisting CD36-positive vasculature. We detected slight upregulation of CD95 by DXR on the resting vessels (unpublished observation). However, no vascular complications or increased endothelial cell apoptosis were observed in these vessels in the course of 35-day treatment, consistent with the observation that quiescent endothelium is more resistant to CD95-mediated apoptosis, ${ }^{32,51-54}$ possibly due to the higher levels of antiapoptotic protein FLIP, ${ }^{55}$ increased stability of Ang1 provided by accessory cells ${ }^{56}$ and other survival signals generated by cell-cell contacts and paracrine factors secreted by surrounding stroma.

Although the effect of DXR/DI-TSP combination in vitro was specific for the endothelial cells, combined treatment caused apoptosis in both endothelial and nonendothelial compartments of the tumor. Such dual in vivo effect is typical for the antiangiogenic therapies and is largely explained by hypoxia and hypoglycemia caused by diminished vascularization. ${ }^{57}$ Recently, a model was proposed where hypoxia-insensitive p53-negative cells are selected during continuous antiangiogenic treatment. ${ }^{58}$ Such p53-negative cells have diminished ability to enter apoptotic program or to undergo cell cycle arrest in response to $\mathrm{HIF}-1 \alpha$ activation. ${ }^{59-61}$ Interestingly, when MTD of DXR were used in conjunction with DI-TSP, the resulting tumors were considerably larger than control-treated ones despite similar decrease in MVD, with the majority of the cells staining negative for p53 (Nelius and Volpert, unpublished observations). Such rapid emergence of the p53negative, hypoxia-resistant cells could be attributed to the extensive DNA damage/repair due to the high genotoxic dose of DXR. Therefore, minimal effective dose of DXR should be considered for the future treatment regimens. 
Recent studies provide another link between TSP1 and chemotherapeutic agents: low-dose metronomic therapy was found to increase TSP1 production and angiogenesis, ${ }^{62}$ while metronomic chemotherapy with cytoxan was shown to decrease circulating endothelial cell progenitors ${ }^{63}$ possibly due to increased TSP1 production. ${ }^{64}$ Here, we demonstrate high antiangiogenic potential of the combination therapy that includes TSP1 antiangiogenic peptide with high specific activity and a metronomic dose of another chemotherapy agent DXR. We show that these agents act synergistically to induce endothelial cell apoptosis and to block angiogenesis and elucidate a mechanism that underlies this synergy. Furthermore, we demonstrate convincingly that the toxic effect of this combination therapy in vivo is extremely low, thus making it an attractive option for a long-term preventative treatment aimed to minimize long-term tumor burden in cancer patients due to residual tumors after primary tumor resection or to the dormant micrometastases.

\section{Experimental Procedures}

\section{Cell culture and reagents}

HUVECs from umbilical cords isolated as described ${ }^{65}$ or purchased from Clonetics (Cambrex BioSciences, Rockland, MD) were maintained as in Lorenzo et al. ${ }^{18}$ HMVECs isolated from neonatal foreskins ${ }^{66-68}$ or purchased from Clonetics were maintained in MCDB131 medium (BioWhittaker, Walkersville, MD, USA) supplemented with $10 \mathrm{mM} \mathrm{HEPES,}$ $15 \%$ FCS, $10 \mathrm{ng} / \mathrm{ml}$ EGF (Promega, Madison, WI, USA), $2.5 \mu \mathrm{g} / \mathrm{ml}$ Fungizone, penicillin, streptomycin, $1 \mu \mathrm{g} / \mathrm{ml}$ hydrocortisone and $2 \mathrm{mM} \mathrm{L-}$ glutamine. Bladder cancer cell line 253J, B-V was maintained in DMEM, $10 \%$ FCS, 2 mM L-glutamine, penicillin and streptomycin. Prostate cancer cells LNCaP were maintained in RPMI medium with $10 \%$ FCS. DXR was from Ferrer Farma (Barcelona, Spain). Recombinant human VEGF was from PeproTech (London, UK). DI-TSP generated as described in Reiher et al. ${ }^{34}$ was generously provided by Abbott Laboratories.

\section{Flow cytometry analysis}

Endothelial cells were plated in $60 \mathrm{~mm}$ gelatinized tissue culture dishes $\left(1 \times 10^{5} /\right.$ plate $)$, treated with indicated DI-TSP/DXR combinations and harvested with trypsin/EDTA. The cells were washed with cold phosphatebuffered saline (PBS), incubated 30 min on ice with anti-CD95 mouse monoclonal antibody DX-2 ( $1 \mu \mathrm{g} / \mathrm{ml}$, PharMingen, San Diego, CA, USA) and $30 \mathrm{~min}$ with FITC-conjugated rabbit anti-mouse IgG (DAKO, 1:50 dilution). Flow cytometry was performed with FACScan (Becton Dickinson) or Cyan Flow (Dako) flow cytometers and the results analyzed using CellQuest or Summit software. The baseline was determined using cells incubated with the secondary antibody alone.

\section{Animal studies}

All animal studies were handled according to the animal care and use federal guidelines approved by the National Institute of Health.

\section{Matrigel plug assay}

Matrigel plug assay was performed as described. ${ }^{69}$ Cold growth factor depleted Matrigel (BD Biosciences) supplemented with 64U/ml Heparin (Sigma, MO, USA) and $250 \mathrm{ng} / \mathrm{ml}$ VEGF was injected into the median abdominal area of anesthetized C57BL6 mice (400 $\mu$ //mouse). To block CD95/CD95L interactions, Matrigel was mixed with CD95 decoy (CD95D) (Sigma, $1 \mu \mathrm{g} / \mathrm{ml})$. To block CD36, anti-CD36 lgA antibody $(5 \mu \mathrm{g} / \mathrm{ml}$, Cascade Bioscience) or IgA isotype control antibody ( $5 \mu \mathrm{g} / \mathrm{ml}$, Southern Biotech) were added. All antibodies were dialyzed to remove $\mathrm{NaN}_{3}$. The plugs solidified at body temperature. DI-TSP was administered daily as intraperitoneal (i.p.) injections and DXR was given i.p. every 5 days. On days 8-14, the plugs were excised, snap-frozen at $-80^{\circ} \mathrm{C}$ in Tissue-Tek OCT compound (Sakura, Torrance, CA, USA), and cut into $5 \mu \mathrm{m}$ sections.

\section{Heterotopic implantation of tumor cells}

At $70-80 \%$ confluence, bladder cancer cells, 253J B-V cells (cell line derived from aggressive carcinoma of the bladder), were transferred for $24 \mathrm{~h}$ in fresh medium, harvested by brief trypsinization and $1 \times 10^{6}$ cells were injected subcutaneously into the hindquarters of athymic mice (nu/ nu), five animals/group, two sites/animal. LNCaP prostate cancer cells were injected into the right flanks (nu/nu mice, 9-10 animals/group) in $200 \mu$ l Matrigel (BD Biosciences), at $2 \times 10^{6}$ cells/site. DI-TSP and DXR treatments were carried out as above. Tumors were measured with a microcaliper every 2 days. At the completion of experiment, the animals were killed, tumors removed, weighted, measured, snap-frozen in OCT compound and processed for analysis.

\section{Immunofluorescence staining}

Cryosections $(5 \mu \mathrm{m})$ of snap-frozen Matrigel plugs or tumor xenografts were fixed in cold acetone, 1:1 acetone/chloroform and cold acetone (5 min/treatment), rinsed in PBS and incubated with Avidin/Biotin Blocking Kit (Vector Laboratories) to block endogenous biotin/avidin. Slides were washed in PBS, blocked 30 min in $2 \%$ donkey serum, $2 \%$ goat serum in PBS and incubated $1 \mathrm{~h}$ with rabbit anti-CD95L $(2.5 \mu \mathrm{g} / \mathrm{ml}$, Calbiochem, San Diego, CA, USA) and $4 \mu \mathrm{g} / \mathrm{ml}$ rat anti-mouse CD31 (Santa Cruz, CA, USA) in blocking solution. The slides were washed in PBS and incubated 30 min with biotinylated anti-rabbit antibodies (1:200 dilution, Vector Laboratories) and donkey anti-rat RhodamineX-conjugated antibodies (1:200 dilution, Jackson Immunoresearch, West Grove, PA, USA) in blocking solution. Slides were developed for $15 \mathrm{~min}$ with FITC-conjugated Fluorescein Avidin D (20 $\mu \mathrm{g} / \mathrm{ml}$, Vector Laboratories). To visualize CD95/ Fas, the sections were reincubated with Biotin/Avidin Kit, washed and blocked with $4 \%$ goat serum in PBS for 30 min followed by $1 \mathrm{~h}$ incubation with anti-CD95 antibody $(2.5 \mu \mathrm{g} / \mathrm{ml}, \mathrm{C}-20$, Santa Cruz Biotech, Santa Cruz, CA, USA) in blocking solution. Biotinylated anti-rabbit antibodies were applied in blocking solution at $1: 200$ dilution for $30 \mathrm{~min}$, the slides washed, exposed for $15 \mathrm{~min}$ to $1 \mu \mathrm{g} / \mathrm{ml}$ Streptavidin Cy5 (Jackson Immunoresearch), dried and mounted using VectaShield Mounting Media (Vector Laboratories).

\section{Microvessel counts}

The tumors fixed as above were blocked in $3 \%$ donkey serum, $1 \% \mathrm{BSA}$ in PBS. The slides were incubated $1 \mathrm{~h}$ (room temperature) with rat antimouse CD31 antibody (PharMingen, San Diego, CA, USA), washed and reblocked. RhodaminX-labeled anti-rat antibodies were added in blocking solution for $1 \mathrm{~h}$, slides washed, and mounted in VectaShield Mounting Medium. Quantitative digital image analysis was performed using MetaView software. We defined MVD as the average area corresponding to the CD31-positive structures counted in at least 10 high-powered $(\times 100)$ field per experimental condition. Statistical significance was evaluated with paired Student's $t$-test. 


\section{Quantification of endothelial CD95 and CD95L expression}

To evaluate CD95 expression in blood vessels, the area of CD31-positive structures was measured using LSM 510 (Zeiss) and Laserpix (BioRad) software. The areas of CD95 and CD95L-positive structures, which colocalized with CD31 staining, were measured and CD95/CD31 and CD95L/CD31 ratios calculated.

\section{Combined staining for CD95L, CD31 and apoptosis}

Staining for CD95L/CD31 was performed as above, the slides were washed and cells positive for DNA fragmentation detected by TUNEL (ApopTag $^{\mathrm{TM}}$ kit, Intergen, Purchase, NY, USA) (this assay allows to discriminate between apoptosis and necrosis). The slides were mounted and fluorescent images analyzed by confocal microscopy. Quantification was performed where indicated, using MetaView software.

For the in situ staining for CD31/apoptosis, HMVECs were plated on gelatinized coverslips, grown to $60 \%$ confluence and treated $24 \mathrm{~h}$ with DXR/DI-TSP combinations. The slides were fixed $10 \mathrm{~min}$ in cold acetone washed with PBS and stained consecutively for CD31 and with ApopTag ${ }^{\mathrm{TM}}$ as above.

Statistical evaluaton of the data was carried out using paired Student's $t$-test and a two-way ANOVA.

\section{Acknowledgements}

This work has been supported by ACS Grant RSG-01-099-01-CSM (OV), $\mathrm{NIH}$ grant R01 HL68003-01 and an award from Abbott Laboratories (OV). JMR was supported by FIS Grant 01/218 and RECAVA from Ministerio de Sanidad y Consumo of Spain and SAF 2003-02920 from DGI, MCYT. AJQ is holder of a FPI fellowship from Ministerio de Ciencia y Tecnología of Spain. AA is supported by a CNIC grant. We are grateful to Drs Zhou Wang (Northwestern University) and Abelardo López-Rivas (Instituto de Parasitología y Biomedicina) for helpful discussion. We want to thank Pilar Torralbo and Alberto Álvarez from CNIC Flow Cytometry Facility.

\section{References}

1. Hanahan D and Folkman J (1996) Patterns and emerging mechanisms of the angiogenic switch during tumorigenesis. Cell 86: 353-364

2. Kerbel $R$ and Folkman $\mathrm{J}$ (2002) Clinical translation of angiogenesis inhibitors. Nat. Rev. Cancer 2: 727-739

3. Rodriguez-Manzaneque JC, Lane TF, Ortega MA, Hynes RO, Lawler J and Iruela-Arispe ML (2001) Thrombospondin-1 suppresses spontaneous tumor growth and inhibits activation of matrix metalloproteinase-9 and mobilization of vascular endothelial growth factor. Proc. Natl. Acad. Sci. USA 98: $12485-12490$

4. Chen H, Herndon ME and Lawler J (2000) The cell biology of thrombospondin1. Matrix Biol. 19: 597-614

5. Dawson DW, Pearce SF, Zhong R, Silverstein RL, Frazier WA and Bouck NP (1997) CD36 mediates the in vitro inhibitory effects of thrombospondin-1 on endothelial cells. J. Cell Biol. 138: 707-717

6. Swerlick RA, Lee KH, Wick TM and Lawley TJ (1992) Human dermal microvascular endothelial but not human umbilical vein endothelial cells express CD36 in vivo and in vitro. J. Immunol. 148: 78-83

7. van Schravendijk MR, Handunnetti SM, Barnwell JW and Howard RJ (1992) Normal human erythrocytes express CD36, an adhesion molecule of monocytes, platelets, and endothelial cells. Blood 80: 2105-2114

8. Tolsma SS, Volpert OV, Good DJ, Frazier WA, Polverini PJ and Bouck N (1993) Peptides derived from two separate domains of the matrix protein thrombospondin-1 have anti-angiogenic activity. J. Cell Biol. 122: 497-511
9. Dawson DW, Volpert OV, Pearce SF, Schneider AJ, Silverstein RL, Henkin J and Bouck NP (1999) Three distinct D-amino acid substitutions confer potent antiangiogenic activity on an inactive peptide derived from a thrombospondin-1 type 1 repeat. Mol Pharmacol. 55: 332-338

10. Jimenez B, Volpert OV, Crawford SE, Febbraio M, Silverstein RL and Bouck N (2000) Signals leading to apoptosis-dependent inhibition of neovascularization by thrombospondin-1. Nat. Med. 6: 41-48

11. Volpert OV, Zaichuk T, Zhou W, Reiher F, Ferguson TA, Stuart PM, Amin M and Bouck NP (2002) Inducer-stimulated Fas targets activated endothelium for destruction by anti-angiogenic thrombospondin-1 and pigment epitheliumderived factor. Nat. Med. 8: 349-357

12. Cardier JE and Erickson-Miller CL (2000) Fas (CD95)- and tumor necrosis factor-mediated apoptosis in liver endothelial cells: role of caspase-3 and the p38 MAPK. Microvasc. Res. 63: 10-18

13. Cardier JE, Schulte T, Kammer H, Kwak J and Cardier M (1999) Fas (CD95, APO-1) antigen expression and function in murine liver endothelial cells: implications for the regulation of apoptosis in liver endothelial cells. FASEB J. 13: $1950-1960$

14. Janin A, Deschaumes C, Daneshpouy M, Estaquier J, Micic-Polianski J, Rajagopalan-Levasseur P, Akarid K, Mounier N, Gluckman E, Socie G and Ameisen JC (2002) CD95 engagement induces disseminated endothelial cell apoptosis in vivo: immunopathologic implications. Blood 99: 2940-2947

15. Krammer PH (1999) CD95(APO-1/Fas)-mediated apoptosis: live and let die. Adv. Immunol. 71: 163-210

16. Krammer PH (2000) CD95's deadly mission in the immune system. Nature 407 : 789-795

17. Herr I and Debatin KM (2001) Cellular stress response and apoptosis in cancer therapy. Blood 98: 2603-2614

18. Lorenzo E, Ruiz-Ruiz C, Quesada AJ, Hernandez G, Rodriguez A, Lopez-Rivas A and Redondo JM (2002) Doxorubicin induces apoptosis and CD95 gene expression in human primary endothelial cells through a p53-dependent mechanism. J. Biol. Chem. 277: 10883-10892

19. Kalyanaraman B, Joseph J, Kalivendi S, Wang S, Konorev E and Kotamraju S (2002) Doxorubicin-induced apoptosis: implications in cardiotoxicity. Mol. Cell. Biochem. 234-235: 119-124

20. Kruh GD and Goldstein LJ (1993) Doxorubicin and multidrug resistance. Curr. Opin. Oncol. 5: 1029-1034

21. Singal PK and lliskovic N (1998) Doxorubicin-induced cardiomyopathy. N. Engl. J. Med. 339: 900-905

22. Lundgren-Eriksson L, Palm A, Ryd W, Warnhammar E and Hultborn R (2001) Radio- and chemotoxicity in mice during hypothermia. Anticancer Res. 21: 3269-3274

23. Abd-Allah AR, Al-Majed AA, Mostafa AM, Al-Shabanah OA, Din AG and Nagi MN (2002) Protective effect of arabic gum against cardiotoxicity induced by doxorubicin in mice: a possible mechanism of protection. J. Biochem. Mol. Toxicol. 16: 254-259

24. Holmes FA and Rowinsky EK (2001) Pharmacokinetic profiles of doxorubicin in combination with taxanes. Semin. Oncol. 28 (Suppl. 12): 8-14

25. Jimenez B and Volpert OV (2001) Mechanistic insights on the inhibition of tumor angiogenesis. J. Mol. Med. 78: 663-672

26. Dinney CP, Fishbeck R, Singh RK, Eve B, Pathak S, Brown N, Xie B, Fan D, Bucana CD and Fidler IJ (1995) Isolation and characterization of metastatic variants from human transitional cell carcinoma passaged by orthotopic implantation in athymic nude mice. J. Urol. 154: 1532-1538

27. Martinez-Irujo JJ, Villahermosa ML, Alberdi E and Santiago E (1996) A checkerboard method to evaluate interactions between drugs. Biochem. Pharmacol. 51: 635-644

28. Komarov PG, Komarova EA, Kondratov RV, Christov-Tselkov K, Coon JS, Chernov MV and Gudkov AV (1999) A chemical inhibitor of p53 that protects mice from the side effects of cancer therapy. Science 285: 1733-1737

29. Beecken WD, Fernandez A, Joussen AM, Achilles EG, Flynn E, Lo KM, Gillies SD, Javaherian K, Folkman J and Shing Y (2001) Effect of antiangiogenic therapy on slowly growing, poorly vascularized tumors in mice. J. Natl. Cancer Inst. 93: 382-387

30. Ferrara N, Hillan KJ, Gerber HP and Novotny W (2004) Discovery and development of bevacizumab, an anti-VEGF antibody for treating cancer. Nat. Rev. Drug Discov. 3: 391-400 
31. Dameron KM, Volpert OV, Tainsky MA and Bouck N (1994) Control of angiogenesis in fibroblasts by p53 regulation of thrombospondin-1. Science 265: $1582-1584$

32. Lawler J (2000) The functions of thrombospondin-1 and-2. Curr. Opin. Cell Biol. 12: $634-640$

33. Volpert OV, Tolsma SS, Pellerin S, Feige JJ, Chen H, Mosher DF and Bouck NP (1995) Inhibition of angiogenesis by thrombospondin-2. Biochem. Biophys. Res. Commun. 217: 326-332

34. Reiher FK, Volpert OV, Jimenez B, Crawford SE, Dinney CP, Henkin J, Haviv F, Bouck N P and Campbell SC (2002) Inhibition of tumor growth by systemic treatment with thrombospondin-1 peptide mimetics. Int. J. Cancer. 98: 668-682

35. Huang H, Campbell SC, Bedford DF, Nelius T, Veliceasa D, Shroff EH, Henkin J, Schneider A, Bouck N and Volpert OV (2004) Peroxisome proliferatoractivated receptor gamma ligands improve the antitumor efficacy of thrombospondin peptide ABT510. Mol. Cancer Res. 2: 541-550

36. Sata M, Suhara T and Walsh K (2000) Vascular endothelial cells and smooth muscle cells differ in expression of Fas and Fas ligand and in sensitivity to Fas ligand-induced cell death: implications for vascular disease and therapy. Arterioscler. Thromb. Vasc. Biol. 20: 309-316

37. Peter ME and Krammer PH (2003) The CD95(APO-1/Fas) DISC and beyond. Cell Death Differ. 10: 26-35

38. Schuler M and Green DR (2001) Mechanisms of p53-dependent apoptosis. Biochem. Soc. Trans. 29: 684-688

39. Manfredi J (2003) p53 and apoptosis: It's not just in the nucleus anymore. Mol. Cell 3: $552-554$

40. Mihara M, Erster S, Zaika A, Petrenko O, Chittenden T, Pancoska P and Moll UM (2003) p53 has a direct apoptogenic role at the mitochondria. Mol. Cell 11: 577-590

41. Nakano K and Vousden KH (2001) PUMA, a novel proapoptotic gene, is induced by $\mathrm{p53}$. Mol. Cell 7: 683-694

42. Liu S, Bishop WR and Liu M (2003) Differential effects of cell cycle regulatory protein p21(WAF1/Cip1) on apoptosis and sensitivity to cancer chemotherapy. Drug Resist. Update 6: 183-695

43. Hahnfeldt $P$, Folkman J and Hlatky L (2003) Minimizing long-term tumor burden: the logic for metronomic chemotherapeutic dosing and its antiangiogenic basis. J. Theor. Biol. 220: 545-554

44. Teicher BA, Emi Y, Kakeji Y and Northey D (1996) TNP-470/minocycline/ cytotoxic therapy: a systems approach to cancer therapy. Eur. J. Cancer. 32A: 2461-2466

45. Jain RK (2001) Normalizing tumor vasculature with anti-angiogenic therapy: a new paradigm for combination therapy. Nat. Med. 7: 987-989

46. Weinstein DM, Mihm MJ and Bauer JA (2000) Cardiac peroxynitrite formation and left ventricular dysfunction following doxorubicin treatment in mice. J. Pharmacol. Exp. Ther. 294: 396-401

47. Evans PC, Taylor ER and Kilshaw PJ (2001) Signaling through CD31 protects endothelial cells from apoptosis. Transplantation 71: 457-460

48. Bird IN, Taylor V, Newton JP, Spragg JH, Simmons DL, Salmon M and Buckley CD (1999) Homophilic PECAM-1(CD31) interactions prevent endothelial cell apoptosis but do not support cell spreading or migration. J. Cell Sci. 112: 1989-1997

49. Newman PJ (1997) The biology of PECAM-1. J. Clin. Invest. 99: 3-8

50. Kotamraju S, Konorev EA, Joseph J and Kalyanaraman B (2000) Doxorubicininduced apoptosis in endothelial cells and cardiomyocytes is ameliorated by nitrone spin traps and ebselen. Role of reactive oxygen and nitrogen species. J. Biol. Chem. 275: 33585-33592

51. Sata M and Walsh K (1998) Oxidized LDL activates fas-mediated endothelial cell apoptosis. J. Clin. Invest. 102: 1682-1689
52. Sata M and Walsh K (1998) TNFalpha regulation of Fas ligand expression on the vascular endothelium modulates leukocyte extravasation. Nat. Med. 4 $415-420$

53. Suhara T, Fukuo K, Sugimoto T, Morimoto S, Nakahashi T, Hata S, Shimizu M and Ogihara T (1998) Hydrogen peroxide induces up-regulation of Fas in human endothelial cells. J. Immunol. 160: 4042-4047

54. Richardson BC, Lalwani ND, Johnson KJ and Marks RM (1994) Fas ligation triggers apoptosis in macrophages but not endothelial cells. Eur. J. Immunol. 24: $2640-2645$

55. Aoudjit F and Vuori K (2001) Matrix attachment regulates Fas-induced apoptosis in endothelial cells: a role for c-flip and implications for anoikis. J. Cell Biol. 152: 633-643

56. Asahara T, Chen D, Takahashi T, Fujikawa K, Kearney M, Magner M, Yancopoulos GD and Isner JM (1998) Tie2 receptor ligands, angiopoietin-1 and angiopoietin-2, modulate VEGF-induced postnatal neovascularization. Circ. Res. 83: 233-240

57. Folkman J (2003) Angiogenesis and apoptosis. Semin. Cancer Biol. 13: $159-167$

58. Yu JL, Coomber BL and Kerbel RS (2002) A paradigm for therapy-induced microenvironmental changes in solid tumors leading to drug resistance. Differentiation 70: 599-609

59. Piret JP, Mottet D, Raes M and Michaiels C (2002) Is HIF-1alpha a pro- or an anti-apoptotic protein? Biochem. Pharmacol. 64: 889-892

60. Graeber TG, Osmanian C, Jacks T, Housman DE, Koch CJ and Lowe SWand Giaccia AJ (1996) Hypoxia-mediated selection of cells with diminished apoptotic potential in solid tumours. Nature 379: 88-91

61. Blagosklonny MV (2001) Hypoxia-inducible factor: Achilles' heel of antiangiogenic cancer therapy (review). Int. J. Oncol. 19: 257-262

62. Bocci G, Francia G, Man S, Lawler J and Kerbel RS (2003) Thrombospondin 1, a mediator of the antiangiogenic effects of low-dose metronomic chemotherapy. Proc. Natl. Acad. Sci. USA. 100: 12917-12922

63. Viloria-Petit A, Miquerol L, Yu JL, Gertsenstein M, Sheehan C, May L, Henkin J, Lobe C, Nagy A, Kerbel RS and Rak J (2003) Contrasting effects of VEGF gene disruption in embryonic stem cell-derived versus oncogene-induced tumors. EMBO J. 22: 4091-4102

64. Bertolini F, Paul S, Mancuso P, Monestiroli S, Gobbi A, Shaked Y and Kerbe RS (2003) Maximum tolerable dose and low-dose metronomic chemotherapy have opposite effects on the mobilization and viability of circulating endothelial progenitor cells. Cancer Res. 63: 4342-4346

65. Munoz C, Castellanos MC, Alfranca A, Vara A, Esteban MA, Redondo JM and de Landazuri MO (1996) Transcriptional up-regulation of intracellular adhesion molecule-1 in human endothelial cells by the antioxidant pyrrolidine dithiocarbamate involves the activation of activating protein-1. J. Immunol. 157: 3587-3597

66. Davison PM, Bensch K and Karasek MA (1980) Isolation and growth of endothelial cells from the microvessels of the newborn human foreskin in cell culture. J. Invest. Dermatol. 75: 316-321

67. Kubota Y, Kleinman HK, Martin GR and Lawley TJ (1988) Role of laminin and basement membrane in the morphological differentiation of human endothelial cells into capillary-like structures. J. Cell Biol. 107: 1589-1598

68. Marks RM, Czerniecki M and Penny R (1985) Human dermal microvascular endothelial cells: an improved method for tissue culture and a description of some singular properties in culture. In Vitro Cell Dev. Biol. 21: 627-635

69. Segura I, Serrano A, De Buitrago GG, Gonzalez MA, Abad JL, Claveria C, Gomez L, Bernad A, Martinez AC and Riese HH (2002) Inhibition of programmed cell death impairs in vitro vascular-like structure formation and reduces in vivo angiogenesis. FASEB J. 16: 833-841

Supplementary Information accompanies the paper on the Cell Death and Differentiation website (http://www.nature.com/cdd) 